\title{
From Bench to Bedside
}

\section{From Bench to Bedside: Alpha-defensin-The Biggest Thing in Joint Replacement Infections Since Prophylactic Antibiotics?}

\author{
Benjamin K. Potter MD
}

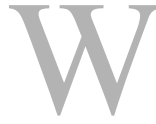

e have been awaiting the arrival of $21^{\text {st }}$ century biomarker-, gene- or sequence-driven personalized medicine

A Note from the Editor-In-Chief:

I am pleased to introduce "From Bench to

Bedside," a new quarterly column written by

Benjamin K. Potter MD. Dr. Potter is a

clinician-scientist in the Department of

Orthopaedics at Walter Reed National

Military Medical Center and in the

Department of Surgery at the Uniformed

Services University of Health Sciences. His

column will investigate important

developments that are making-or are about

to make - the transition from the laboratory to clinical practice, as well as technologies and approaches that have recently made that jump. The author certifies that he, or any member of his immediate family, has no funding or commercial associations (eg, consultancies, stock ownership, equity interest, patent/licensing arrangements, etc) that might pose a conflict of interest in connection with the submitted article.

All ICMJE Conflict of Interest Forms for authors and Clinical Orthopaedics and Related Research ${ }^{\mathbb{R}}$ editors and board members are on file with the publication and can be viewed on request.

The opinions expressed are those of the writers, and do not reflect the opinion or policy of CORR $^{\circledR}$ or the Association of Bone and Joint Surgeons ${ }^{\mathbb{R}}$.

The author is an employee of the US

Government and this work was prepared as part of his official duties. As such, there is no since well before the millennium. Yet the "Golden Age" of personalized medicine has proven elusive. While total joint arthroplasty (TJA) alleviates

copyright to transfer. The views expressed in this article are those of the author and do not necessarily reflect the official policy or position of the Department of the Army, Department of the Navy, Department of Defense, nor the U.S. Government. I certify that all individuals who qualify as authors have been listed; each has participated in the conception and design of this work, the analysis of data, the writing of the document, and the approval of the submission of this version; that the document represents valid work; that if I used information derived from another source, I obtained all necessary approvals to use it and made appropriate

acknowledgements in the document; and that each takes public responsibility for it. Nothing in the presentation implies any Federal/DOA/DON/ DOD endorsement. The author received no financial support for this editorial.

B. K. Potter MD $(\varangle)$

Department of Orthopaedics, Walter

Reed National Military Medical Center, 8901 Wisconsin Ave, America Building (19), 2nd Floor - Ortho, Bethesda, MD 20889, USA

e-mail: Benjamin.k.potter.mil@mail.mil

B. K. Potter MD

Department of Surgery, Uniformed

Services University of Health Sciences, Bethesda, MD, USA suffering perhaps better than any other orthopaedic intervention [8], patients whose arthroplasties have been complicated by infection have a very different experience. The condition is painful, the treatments are inconsistent, and most vexingly, our approaches to diagnosing the condition fall well short of what our patients expect from us in the second decade of the $21^{\text {st }}$ century.

Complications and failures outcomes remain. Apart from rare fatal pulmonary emboli, which unfortunately require no treatment, periprosthetic joint infection (PJI) remains among the most dreaded complications that our patients experience. Infection rates have dropped in a range of $300 \%$ to $500 \%$ since the days of Charnley's pioneering work in the hip, but PJI remains a devastating and feared complication $[3,10]$. While most infections can be successfully treated by a variety of approaches that stop short of the last resort, alternatives of fusion, resection arthroplasty, or amputation, PJIs continue to be expensive, profoundly impact patient quality of life, and literally kill people [14].

Often, however, the diagnosis is unclear despite routine inflammatory 
laboratory values, radiologic studies, and aspirate fluid and culture analysis. There is little doubt that the (at least two-fold) higher infection rate for revision procedures is not simply a result of the increased duration, complexity, and (immunosuppressive) transfusion requirements of these procedures-many "aseptic" revisions, are not. Excellent work has been performed on aspirate cell analysis, but these results require complex interpretation that varies by time from the index procedure, the joint involved, a "clean" versus "bloody" tap, and even the laboratory normative values at your institution [2, 12]. Serum inflammatory markers are generally afflicted by these same limitations, as well as others, and often perform worse. Cut-off values, while helpful, remain imperfect, seemingly moving targets that (needless to say) have not been calibrated to your specific patient and their potential confounding factors.

To help clarify matters, two excellent (and similar) consensus statements from the Musculoskeletal Infection Society and an international panel $[11,13]$ regarding the diagnosis of periprosthetic joint infections have been produced. While they do not answer all of our questions, these guidelines, at the very least, establish a reference standard we can use to confirm a diagnosis of infection and to measure new diagnostics and therapeutics against.
Ultrasensitive molecular diagnostics have been developed concurrently, but these techniques suffer from expense, lack of widespread adoption outside of clinical investigations, and perhaps from being too sensitive. For example, polymerase chain reaction-based techniques can be limited by both contamination and coincidental findings - a positive finding unfortunately does not necessarily correlate with clinical relevance [9].

Finally, a better answer for the diagnosis of PJI following TJA appears to have arrived in the form of synovial fluid alpha-defensin levels. Measuring synovial fluid levels of this marker, particularly in the context of synovial C-reactive protein, has demonstrated impressive accuracy in the diagnosis of PJI. One series of 26 PJIs and 66 aseptic joints found that $\alpha$-defensin, as well as a few other candidate biomarkers, demonstrated $100 \%$ sensitivity and specificity for the diagnosis of PJI [7]. In a subsequent analysis of 149 synovial aspirates, including 37 with PJI, synovial $\alpha$-defensin demonstrated $97 \%$ sensitivity and $96 \%$ specificity for the diagnosis of PJI, improving to $100 \%$ specificity with interpreted concurrently with synovial C-reactive protein [6]. Most recently, a separate group of investigators found sensitivity of $100 \%$ and specificity of $95 \%$ in a prospective cohort of 57 patients [1]. These findings are all the more important given that only around $50 \%$ of preoperative synovial cultures are typically positive, even in the setting of PJI.

Two additional factors are important when interpreting these promising results. First, these studies included patients with current antibiotic administration and/or systemic inflammatory arthropathies - two factors that frequently frustrate clinicians and obfuscate routine laboratory result interpretation. Second, rather than being stuck, "on the horizon," this test is commercially available now. We can hope that even if we have not yet solved the problem of PJI, we may at least be on the way to a reliable approach to diagnosis.

In closing, full disclosure: Naturally, much of this progress has been driven by "industry" — no implant or test is free, and no such test or device is likely to reach the market if it were. Much of the work to date on $\alpha$ defensin has been done by Dr. Carl Deirmengian and colleagues, who have disclosed their financial relationships in their various publications. Dr. Deirmengian began investigating the host response as a means to better diagnose infections when he was a resident, demonstrating critical differences in neutrophil responses between infection and gout in a Mark Coventry Award winning paper [4]. Ultimately, he determined that the gene chips utilized in this analysis were likely to 


\section{From Bench to Bedside}

impractical for clinical use. However, as a fellow, he revisited the issue of utilizing synovial fluid analysis to diagnose infection. This time focusing on proteins; the resulting manuscript produced promising work that would ultimately spawn his further investigation of $\alpha$ defensin [5], the eventual formation of a company, and commercial product development. More disclosure: Around a decade ago, Dr. Deirmengian and I both competed in a resident research competition. Carl won.

\section{References}

1. Bingham J, Clarke H, Spangehl M, Schwartz A, Beauchamp C, Goldberg B. The alpha defensing-1 biomarker assay can be used to evaluate the potentially infected total joint arthroplasty. Clin Orthop Relat Res. 2014;472:4006-4009.

2. Chalmers PN, Sporer SM, Levine BR. Correlation of aspiration results with periprosthetic sepsis in revision total hip arthroplasty. J Arthroplasty. 2014; 29:438-442.

3. Charnley J. Postoperative infection after total hip replacement with special reference to air contamination in the operating room. Clin Orthop Relat Res. 1972;87:167-187.

4. Deirmengian C, Lonner J, Booth RE Jr. The Mark Coventry Award:
White blood cell gene expression: A new approach toward the study and diagnosis of infection. Clin Orthop Relat Res. 2005;440:38-44.

5. Deirmengian C, Hallab N, Tarabishy A, Della Valle C, Jacobs JJ, Lonner J, Booth RE Jr. Synovial fluid biomarkers for periprosthetic infection. Clin Orthop Relat Res. 2010;468: 2017-2023.

6. Deirmengian C, Kardos K, Kilmartin P, Cameron A, Schiller K, Parvizi J. Combined measurement of synovial fluid alpha-defensin and C-reactive protein levels: Highly accurate for diagnosis periprosthetic joint infection. J Bone Joint Surg Am. 2014;96: 1439-1435.

7. Deirmengian C, Kardos K, Kilmartin P, Cameron A, Schiller K, Parvizi J. Diagnosing periprosthetic joint infection: Has the era of the biomarker arrived? Clin Orthop Relat Res. 2014;472:3254-3262.

8. Ethgen O, Bruyere O, Richy F, Dardennes C, Reginster J-Y. Healthrelated quality of life in total hip and total knee arthroplasty: A qualitative and systematic review of the literature. J Bone Joint Surg Am. 2004;86:963-974.

9. Hartley JC, Harris KA. Molecular techniques for diagnosing prosthetic joint infections. J Antimicrob Chemother. 2014;69 Suppl 1:i21-i24.

10. Kurtz SM, Lau E, Schmier J, Ong KL, Zhao K, Parvizi J. Infection burden for hip and knee replacement arthroplasty in the United States. $J$ Arthroplasty. 2008;23:984-991.

11. Parvizi J, Zmistowski B, Berbari EF, Bauer TW, Springer BD, Della Valle CJ, Garvin KL, Mont MA, Wongworawat MD, Zalavras CG. New definition for periprosthetic joint infection: from the workgroup of the Musculoskeletal Infection Society. Clin Orthop Relat Res. 2011;469: 2992-2994.

12. Yi PH, Cross MB, Moric M, Sporer SM, Berger RA, Della Valle CJ. The 2013 Frank Stinchfield Award: Diagnosis of infection in the early postoperative period after total hip arthroplasty. Clin Orthop Relat Res. 2014;472:424-429.

13. Zmistowksi B, Karam JA, Durinka JB, Casper DS, Parvizi J. Periprosthetic joint infections increase the risk of one-year mortality. J Bone Joint Surg Am. 2013;95:2177-2184.

14. Zmistowski B, Della Valle C, Bauer TW, Malizos KN, Alavi A, Bedair H, Booth RE, Choong P, Deirmengian $\mathrm{C}$, Ehrlich GD, Gambir A, Huang R, Kissin Y, Kobayashi H, Kobayashi N, Krenn V, Lorenzo D, Marston SB, Meermans G, Perez J, Ploegmakers JJ, Rosenberg A, Simpendorfer $\mathrm{C}$, Thomas $\mathrm{P}$, Tohtz $\mathrm{S}$, Villafuerte JA, Wahl P, Wagenaar FC, Witzo E. Diagnosis of periprosthetic joint infection. J Arthroplasty. 2014;29:77-83. 